\title{
The Spread of Infectious Disease on Network Using Neutrosophic Algebraic Structure
}

\author{
A. Zubairu1, A. A. Ibrahim² \\ ${ }^{1}$ Shehu Shagari College of Education, Sokoto, Nigeria \\ ${ }^{2}$ Usmanu Dan Fodiyo University, Sokoto, Nigeria \\ Email:Zubairuabdul44@yahoo.com
}

How to cite this paper: Zubairu, A. and Ibrahin, A.A. (2017) The Spread of Infectious Disease on Network Using Neutrosophic Algebraic Structure. Open Journal of Discrete Mathematics, 7, 77-86. https://doi.org/10.4236/ojdm.2017.72009

Received: November 28, 2016

Accepted: April 21, 2017

Published: April 24, 2017

Copyright ( 92017 by authors and Scientific Research Publishing Inc. This work is licensed under the Creative Commons Attribution International License (CC BY 4.0).

http://creativecommons.org/licenses/by/4.0/

\section{cc) (i) Open Access}

\begin{abstract}
Network theory and its associated techniques has tremendous impact in various discipline and research, from computer, engineering, architecture, humanities, social science to system biology. However in recent years epidemiology can be said to utilizes these potentials of network theory more than any other discipline. Graph which has been considered as the processor in network theory has a close relationship with epidemiology that dated as far back as early 1900 [1]. This is because the earliest models of infectious disease transfer were in a form of compartment which defines a graph even though adequate knowledge of mathematical computation and mechanistic behavior is scarce. This paper introduces a new type of disease propagation on network utilizing the potentials of neutrosophic algebraic group structures and graph theory.
\end{abstract}

\section{Keywords}

Neutrosophic Group $N(G)$, Infectious Disease, Graph Representation of $N(G)$, Cayley Graphs

\section{Introduction}

Mathematical models is being used in studying the dynamics, transfer and control of infectious disease. Most of the epidemiological models are continuous time models which uses ordinary differential equation [2] and assumed that the population is fixed and well mixed and the transmission is evaluated over period of time. However the environment in which the population lives is often heterogeneous, therefore there is need to distinguish between the locations. There is closed relationship between epidemiology and graph theory that dated as far back as early 1900. This is because compartments, groupings and household 
models are created and used to study dynamics of epidemics even though mathematical and mechanistic knowledge is scarce. More recently graphs and trees are being used to study the dynamics of infectious disease. In this type, nodes represents infected agents and arc represents transmission from one agent to another. During the review of the literature we found out that most of the authors used compartment-based modelling in the transmission of the pathogens. In order to demonstrate how the population moves through, during the course of the epidemic processes, different compartment were formulated. Common among them are SIR, SIS and SEIR. In most of these models, two basic assumptions were often made so as to simplify the modelling process: 1) uniform mixing assumption and 2) homogeneity assumption, both of which may not yield a good result. We also found out that considerable importance is placed on analytical approaches and statistical methods which are both rapidly expanding fields. We note that less attention is paid to the algebraic structures, therefore we intend to explore a novel approach with the algebraic graph theory and the neutrosophic algebraic structure first introduce by [3]. This is a new branch of philosophy which actually studies the origin, nature, and scope of neutralities. This theory is being applied in many fields in order to solve problems related to indeterminacy.

In this paper we represent each stage of the viral transmission with element of $Z_{7}$, where each stage is accorded with a unique element in $Z_{7}$. Therefore we are able to distinguish between those who are unable to catch the infection with those susceptible. Moreover we noticed that the infected population were counted as one entity in all of those models, so that transmission from infected class to the next compartment raises a lot of questions. Here these shortcomings were fully taken care of by splitting the infected class into two categories, i.e., Infected vs transmitter. We are focusing on the neutrosophic graphs created by [4], because of the indeterminacy in some nodes and edges which distinguishes it from the classical graph theory. So we can label each point of the viral transmission stages with the notation $a+b I$ where $a, b \in \mathbb{R}, \mathbb{Z}$, or $\mathbb{C}$. Here $N(G)=\left\{\left\langle Z_{7} \cup I\right\rangle,{ }^{\prime}+\right.$ 'modulo 7$\}$ is use and we found that this neutrosophic group is also a cyclic group modulo 7. But since $N(G)$ is a union of elements of $Z_{7}$ and the indeterminacy " $P$ " then there is no single generator for the group, instead a disjoint class of generators with a maximum of five (5) elements each. So what we are present here is a transformation of the representation of different stages of the viral transmission into neutrosophic group structure thereby constructing neutrosophic graphs from this group.

In many disease transmission models it is unfeasible to exactly retrieve the complete network structure but with neutrosophic integer modulo this shortcoming is overcome because we enlarge the graph so as to accommodate more nodes.

\section{Basic Definition}

Feinleib defines epidemiology as "the study of the distribution and determinants of health related states or events in specified populations, and the application of 
this study to control of health problems" [5].

Epidemiology is defined by Kramer as "it is a scientific method which can be applied to a broad range of health and medical problems, from infectious diseases to health care" [6].

When referring to disease spread various terminologies are used, such as:

1) Disease Outbreak: a disease outbreak occurs when there is a sudden increase in the expected number of affected people.

2) Epidemic: during a disease outbreak, when the incidence rate increases above the previous occurrences of the outbreak it is known as epidemic.

3) Pandemic: epidemic affecting a large population is known as pandemic.

4) Endemic: if the epidemic remains within a particular community, it is called endemic [7].

Stages of Infection:

The infection has different stages in a person and the duration and strength vary according to a person's immunity, age, health, previous exposure to the infection etc. A person who is not infected and has a chance of getting infected is said to be in susceptible stage. A person's immunity system may destroy the infectious organism. It may continue to stay inside a person's body depending upon his characteristics. Different stages of infection are as follows:

- Latent Period

The latent period is the period between the start of infection to the infectious period.

- Incubation Period

It is the time period between the infectious period and the onset of symptoms.

- Symptomatic Period

Symptomatic period starts after the incubation period when the symptoms show up till the infected person stops infecting other.

The stages of infection summarized by Nelson as shown in Figure 1 which is grouped into Susceptible, infected, infective and removed. It shows the level of agent in host from the start of the infection and increase instantaneously over time until it reaches its pick and then dies out momentarily.

Although Nelson was able to distinguish between infected and infective period that is a person might be infected but yet not infectious until later in life, what is lacking in his model is the category of infected individuals who will never be infectious but rather will remain in the infected class until been recovered. Our proposed model is set to capture this targeted population together with the category of those not susceptible members of the population whom might have significant effects in determining the epidemic threshold and other epidemiological concepts.

Neutrosophic group:

Let $(G, *)$ be any group, the neutrosophic group is generated by $I$ and $G$, under * denoted by $N(G)=\{\langle G \cup I\rangle, *\} \quad[9]$.

Where " $P$ " is the indeterminate and,

$I^{2}=I, I+I+I \cdots n$ times $=n I I-I=0,0 I=0$. 


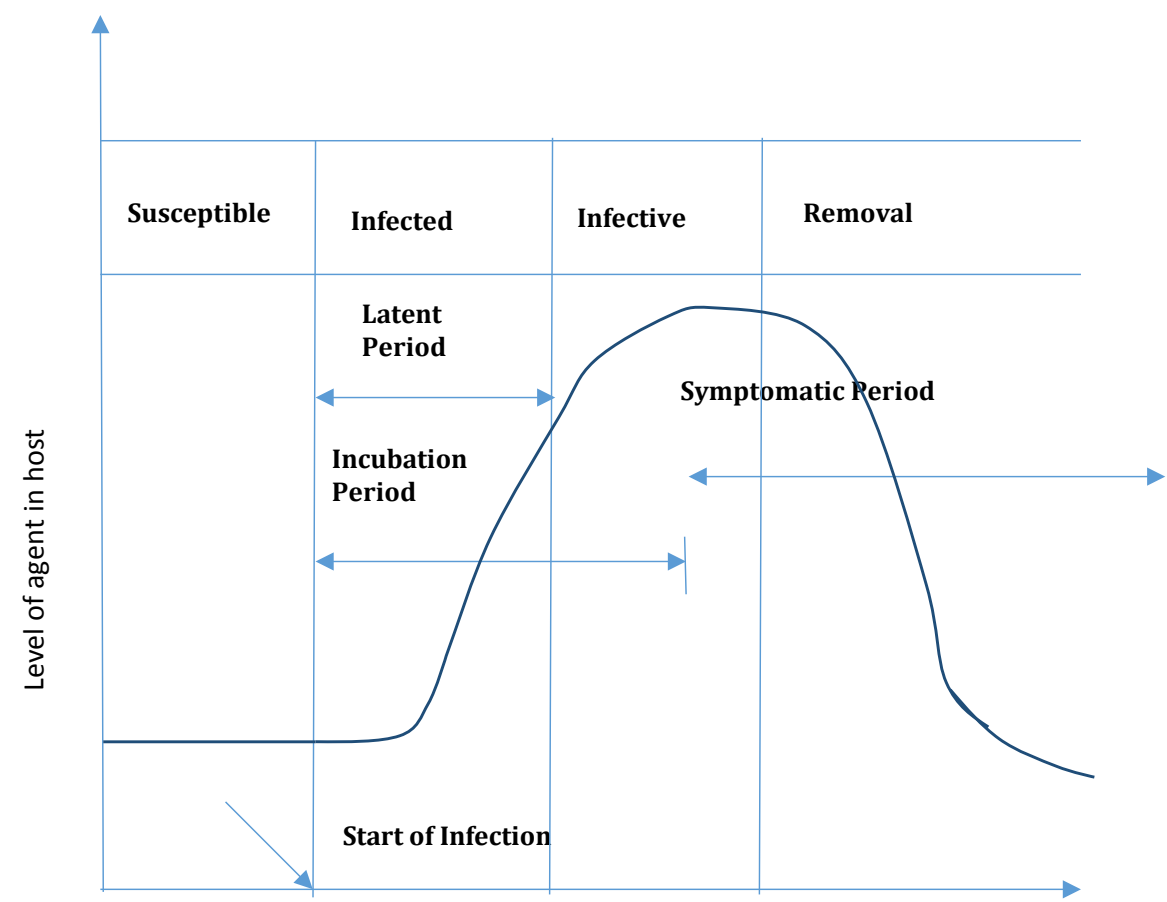

Figure 1. Stages of infection [8].

It is however to note that:

1) $N(G)$ in general is not a group by itself.

2) $N(G)$ always contains a group [10].

Form this definition, however by defining

$\left\{\left\langle Z_{7} \cup I\right\rangle\right.$, '+'modulo 7$\}=\left\{a+b I / a, b\left(b \in Z_{7}\right)\right\}$, then $N(G)$ can be made a group.

Graph:

We know that a graph $G$ consists of two things:

1) $A$ set $V$ of elements called nodes (or points or vertices).

2) $A$ set $E$ of edges such that each edge in $E$ is identified with a unique (unordered) pair $\{u, v\}$ of nodes in $V$, denoted by $e=\{u, v\}$. So $G=(V, E)$. So from the fundamental concepts on graph theory and the knowledge of neutrosophic group we can construct a neutrosophic graph which will serve as our viral transmission model.

From the review of literature we discovered that the first representation of groups by graphs is the Cayley graphs. These graphs were introduced by Arthor Cayley in 1878 and it shows pictorial representations of finite groups [11].

Cayley graph:

Giving a group $A$ and a set of generators for $A$. The Cayley digraph $G=(V, E)$ denoted by $\langle A, S\rangle$ is constructed as follows:

1) The elements of the group $A$ forms the vertices $V$ of the digraph $G$.

2) The edge $(a, b)$ is in $E$ if and only if $a g=b$ for some generators $g$ in $S$.

If $S=S \cup S^{\prime}$, then $G$ is Cayley graph [12] [13].

$G$ graph:

For a group $G$ with generating set $S=\left\{s_{1}, s_{2}, \cdots s_{k}\right\}$, the $G$-graph of $G$, de- 
noted by $\Gamma(G, S)$ is the graph whose vertices are distinct cosets of $\left\langle s_{i}\right\rangle$ in $G$. Two distinct vertices are joined by an edge when the set intersection of the cosets is nonempty [14].

Combining the above two definitions, Cayley graph and $G$-graph it is evidently clear that:

The generators of a cyclic group plays a vital role in constructing a graph from group and there is a relationship between a generator and its generating set which can be considered to be an arc, whether directed or undirected.

\section{Methodology}

\section{1) Assumptions:}

a) The total population is taken as a constant.

b) An initial infected class is introduced to the total population.

c) The dead or recovered people in the community belongs to the same class.

d) The population's contact is heterogeneous.

2) Variables Declaration:

$S^{*}=$ Not Susceptible, represents those members of the population who are negated from the disease or whose probability of being contracted with the disease is zero.

$S=$ Susceptible, represents those members of the population who stand the risk of being infected.

$L=$ Incubation, represents those members of the population who are infected and the body's defence mechanisms are unable to destroy the virus.

$E=$ Exposed, This represents the period when the symptoms start to shows up.

$I=$ Infected, This represents a fraction of the population who are infected and remain in the infected class but potentially cannot transmit the disease. From Figure 2 this indicates that the curve increase in the infected class and then drops without touching the infective class. Epidemiologically this means it remains stagnant in the infected class, until being removed or return to susceptible.

$T=$ Transmitter, This class of the population are infected and are capable of transmitting the infection.

$R=$ Recovered, These are fraction of the population, that are immune, vaccinated, dead, isolated.

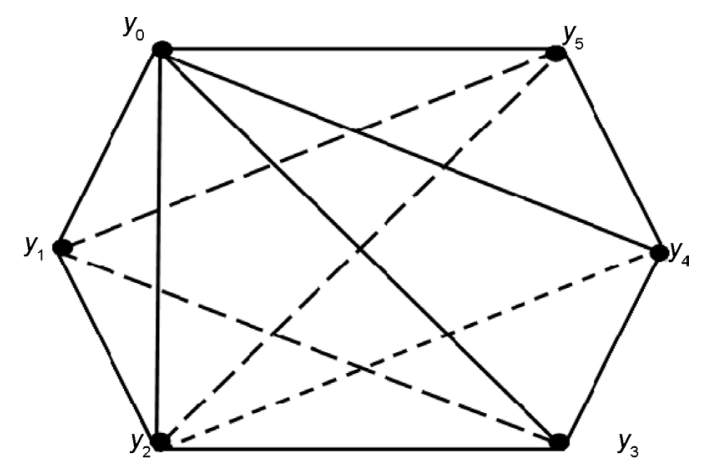

Figure 2. Neutrosophic viral transmission graph. 
$G=$ Represents the total population. In this case $S, L, E, I, T, R, S^{*}$ are subset of $G$.

Hence $\left(S, L, E, I, T, R, S^{*}\right) \subset G$.

Let us use the integer modulo arithmetic $Z_{n}$ to represents the subsets of $G$, that is

$$
S^{*}=0, S=1, L=2, E=3, I=4, T=5, R=6 .
$$

Observed that $G=\{0,1,2,3,4,5,6\}$. Consider the elements of $G$ to be integer modulo 7 hence $\left(Z_{7}+\right)$ is a group.

Lets define $N(G)=\left\{\left\langle Z_{7} \cup I\right\rangle\right.$, '+'modulo 7$\}$ is a neutrosophic group which is in fact a group. For $N(G)=\left\{a+b I / a, b \in\left(Z_{7}\right)\right\}$ is a group under "+" modulo 7. Thus this neutrosophic group is also a group.

Hence

$$
N(G)=\left\{\begin{array}{c}
0,1,2,3,4,5,6, I, 2 I, 3 I, 4 I, 5 I, 6 I, 1+I, 1+2 I, 1+3 I, 1+4 I, 1+5 I, \\
1+6 I, 2+I, 2+2 I, 2+3 I, 2+4 I, 2+5 I, 2+6 I, 3+I, 3+2 I, 3+3 I, \\
3+4 I, 3+5 I, 3+6 I, 4+I, 4+2 I, 4+3 I, 4+4 I, 4+5 I, 4+6 I, 5+I \\
5+2 I, 5+3 I, 5+4 I, 5+5 I, 5+6 I, 6+I, 6+2 I, 6+3 I, 6+4 I, \\
6+5 I, 6+6 I .
\end{array}\right\}
$$

Here " $P$ " cannot stand for the group identity it is only an indeterminate and hence suitable for the nature of our disease propagation model.

Theorems:

Hence the following theorems were proposed based on the group $N(G)$.

Theorem (1) Every stage of viral transmission is allocated a unique representation in $N(G)$.

Proof: Suppose we have two representation for $S$ i.e. $s, s^{\prime} \in S$.

If $e \in N(G)$ is the identity element and be such that has no effect on the elements of $N(G)$, then $e^{*} s=s^{*} e=s$ since $e$ is the identity element.

Also $s^{\prime *} e=e^{*} s^{\prime}=s^{\prime}$ since e is the identity element.

Hence $s=s^{\prime}$ and the result follows.

Theorem (2) The operation * on $N(G)$, is well define.

Let $a, b \in N(G)$. By the definition of * on $N(G)$, we would have $a^{*} b=\overline{a+b}$. Now suppose $a_{1} \in a$ and $b_{1} \in b$, be chosen as class representative of $a$ and $b$ respectively by defining a suitable homomorphism on $N(G)$, in this case we would have $a+b=a_{1}+b_{1}=\overline{a_{1}+b_{1}}$. Thus we can show that $\overline{a_{1}+b_{1}}=\overline{a+b}$.

Now $a_{1} \in a \Rightarrow a_{1} \equiv a(\bmod n) \Rightarrow a_{1}=a+k_{n}$ for some $k \in Z$, and $b_{1} \in b \Rightarrow b_{1} \equiv b(\bmod n) \Rightarrow b_{1}=b+h_{n}$ for some $h \in Z$, thus $a_{1}+b_{1}=a+b+(k+h) n \Rightarrow a_{1}+b_{1} \equiv a+b(\bmod ) n$, this implies that $a * b=\overline{a+b}$ and the operation * on $N(G)$, is well define.

Theorem (3) The order of the neutrosophic group $\left\{\left\langle Z_{n} \cup I\right\rangle\right.$,'+'modulo $\left.n\right\}$ denoted by $|N(G)|$ is $n^{2}$.

Proof: Since $Z_{n}$ has $n$ elements, and the indeterminate " $P$ " is such that: $I+I+\cdots n$ times is $n I$ i.e. $|n I|$ is $n$. Therefore $\left\langle Z_{n} \cup I\right\rangle$ is a combinations of the elements of $Z_{n}$ and $n I$ that is $n \times n=n^{2}$ hence the proof. 
3) Building graphs from neutrosophic group structure.

Kandasamy W and Smarandache F in 2015 defines neutosophic graphs as follows: "If the edge values are from the set $R \cup I$ or $Q \cup I$ or $Z_{n} \cup I$ or $Z \cup I$ or $C \cup I$ they are term as neutrosophic graph" [4]. In our own case the edge are from $\left\{\left\langle Z_{7} \cup I\right\rangle\right.$, ,'modulo 7$\}$ and the elements of $\left(Z_{7}\right)$ where carefully allocated with a unique stages of the viral transmission. Now lets define a neutrosophic graph generated from neutrosophic group.

Definition: Henceforth we defined neutrosophic graph $\varnothing(V, E)$ generated from $N(G)$ as follows:

1. Given an element $a \in N(G)$ and generates $a$ set $B$ of elements in $N(G)$ then element $a$ and set $B$ connects or has a relation. Hence For any $b \in B,(a, b)$ is an edge.

2. The elements of $N(G)$ forms the vertices $V$ of the neutrosophic graph.

As mentioned above and confirmed by Dinnen and Breto the generator $(s)$ of a group $G$ is very vital in the construction of a neutrosophic graph from group.

4) Finding set of generators

Since the neutrosophic group $N(G)$, is generated by $Z_{7} \cup I$, the generators are precisely the class of union of generators which partitioned $N(G)$.

Hence in $Z_{7}$ the generators are $(1,2,3,4,5,6)$. Moreover

$I$ is a generator of $2 I, 3 I, 4 I, 5 I, 6 I$,

$1+I$ is a generator of $2+2 I, 3+3 I, 4+4 I, 5+5 I, 6+6 I$,

$1+2 I$ is a generator of $2+4 I, 3+6 I, 4+I, 5+3 I, 6+5 I$,

$1+3 I$ is a generator of $2+6 I, 3+2 I, 4+5 I, 5+I, 6+4 I$,

$1+4 I$ is a generator of $2+I, 3+5 I, 4+2 I, 5+6 I, 6+3 I$,

$1+5 I$ is a generator of $2+3 I, 3+I, 4+6 I, 5+4 I, 6+2 I$,

$1+6 I$ is a generator of $2+5 I, 3+4 I, 4+3 I .5+2 I, 6+I$,

$2+I$ is a generator of $4+2 I, 6+3 I, 1+4 I, 3+5 I, 5+6 I$,

$2+2 I$ is a generator of $4+4 I, 6+6 I, 1+I, 3+3 I, 5+5 I$,

$2+3 I$ is a generator of $4+6 I, 6+2 I, 1+5 I, 3+I 5+4 I$,

$2+4 I$ is a generator of $4+I, 6+5 I, 1+2 I, 3+6 I, 5+3 I$,

$2+5 I$ is a generator of $4+3 I, 6+I, 1+6 I, 3+4 I, 5+2 I$,

$2+6 I$ is a generator of $4+5 I, 6+4 I, 1+3 I, 3+2 I, 5+I$,

$3+I$ is a generator of $6+2 I 2+3 I, 5+4 I, 1+5 I, 4+6 I$,

$3+2 I$ is a generator of $6+4 I, 2+6 I, 5+I, 1+3 I, 4+5 I$,

$3+3 I$ is a generator of $6+6 I, 2+2 I, 5+5 I, 1+I, 4+4 I$,

$3+4 I$ is a generator of $6+I, 2+5 I, 5+2 I, 1+6 I, 4+3 I$,

$3+5 I$ is a generator of $6+3 I, 2+I, 5+6 I, 1+4 I, 4+2 I$,

$3+6 I$ is a generator of $6+5 I, 2+5 I, 5+3 I, 1+2 I, 4+I$,

$4+I$ is a generator of $1+2 I, 5+3 I, 2+4 I, 6+5 I, 3+6 I$,

$4+2 I$ is a generator of $1+4 I, 5+6 I, 2+I, 6+3 I, 3+5 I$,

$4+3 I$ is a generator of $1+6 I, 5+2 I, 2+5 I, 6+I, 3+4 I$,

$4+4 I$ is a generator of $1+I, 5+5 I, 2+2 I, 6+6 I, 3+3 I$,

$4+5 I$ is a generator of $1+3 I, 5+I, 2+6 I, 6+4 I, 3+2 I$,

$4+6 I$ is a generator of $1+5 I, 5+4 I, 2+3 I, 5+2 I, 3+I$, 
$5+I$ is a generator of $3+2 I, 1+3 I, 6+4 I, 4+5 I, 2+6 I$,

$5+2 I$ is a generator of $3+4 I, 1+6 I, 6+I, 4+3 I, 2+5 I$,

$5+3 I$ is a generator of $3+6 I, 1+2 I, 6+5 I, 4+I, 2+4 I$,

$5+4 I$ is a generator of $3+I, 1+5 I, 6+2 I, 4+6 I, 2+3 I$,

$5+5 I$ is a generator of $3+3 I, 1+I, 6+6 I, 4+4 I, 2+2 I$,

$5+6 I$ is a generator of $3+5 I, 1+4 I, 6+3 I, 4+2 I, 2+I$,

$6+I$ is a generator of $5+2 I, 4+3 I, 3+4 I, 2+5 I, 1+6 I$,

$6+2 I$ is a generator of $5+4 I, 4+6 I, 3+I, 2+3 I, 1+5 I$,

$6+3 I$ is a generator of $5+6 I, 4+2 I, 3+5 I, 2+I, 1+4 I$,

$6+4 I$ is a generator of $5+I, 4+5 I, 3+2 I, 2+6 I, 1+3 I$,

$6+5 I$ is a generator of $5+3 I, 4+I, 3+6 I, 2+4 I, 1+2 I$,

$6+6 I$ is a generator of $5+5 I, 4+4 I, 3+3 I, 2+2 I, 1+I$,

\section{Discussion}

From the result so far generated above we can see that every element $a+b I$ in $N(G)$ partitioned $N(G)$ into subset which are themselves groups each when the identity $0+0 I$ is embedded in it. Therefore a neutrosophic group $N(G)$ is cyclic if for any $a+b I \in\left(Z_{n} \cup \mathrm{I}\right)$, there exist an integer $r \in Z_{n}$ such that $r(a+b I)=c+d I$ for some $c+d I \in N(G)$. Then $a+b I$ is a generator of $c+d I$.

Observed that the principal generators denoted by $\left\{\left\langle p_{g}\right\rangle\right\}$ are those point in $N(G)$ whose generating cycles are distinct. Also the sub generators are those who are either subset of $\left\{\left\langle p_{g}\right\rangle\right\}$ or parts of its generating cycles have been generated by $\left\{\left\langle p_{g}\right\rangle\right\}$.

Corollary If $\left\{\left\langle Z_{n} \cup I\right\rangle,{ }^{\prime}+\right.$ 'modulo $\left.n\right\}$ is a cyclic neutrosophic group and $\langle a\rangle$ is a generator, then it generates only $n-2$ elements.

Proof: The generalization of $N(G)$ as a group is false, but $N(G)$ always contained a group.

Let $Z_{n}$ be the cyclic group in $N(G)$ by the theorem "let $G=\left\langle Z_{n}\right\rangle$ be a finite cyclic group of order $n$. The generators of $G$ are the elements $r a$ where the $\operatorname{gcd}(r, n)=1$ coprime or relatively prime".

Now $r$ is taking from $\left\langle Z_{7}\right\rangle$ where $r=(0,1,2,3,4,5,6), \operatorname{gcd}(0,7)=1$ is impossible, $\operatorname{gcd}(1,7)=1$ Obvious.

Hence each generator, generate only $n-2$ elements in $N(G)$.

Results

Hence given that $y_{0}=\langle 1+I\rangle$, is a generator with its generating sets as $y_{1}=2+2 I, y_{2}=3+3 I, y_{3}=4+4 I, y_{4}=5+5 I, \quad y_{5}=6+6 I$, then the nodes are $V=\left(y_{0}, y_{1}, y_{2} y_{3}, y_{4}, y_{5}\right)$. The dotted lines shows neutrosophic edges, indicating that the connections between those distinct nodes are indeterminate as shown in Figure 2.

Since $y_{0}$ is the generator, then connections to any of its generating sets is guarantee and the tendency of contracting is high, meaning it has a direct communication to those neighbors. But $y_{1}$ to $y_{3}, y_{2}$ to $y_{5}, y_{4}$ to $y_{2}$ all has indeterminate connections, this indicates that the tendencies of contraction is vague. 


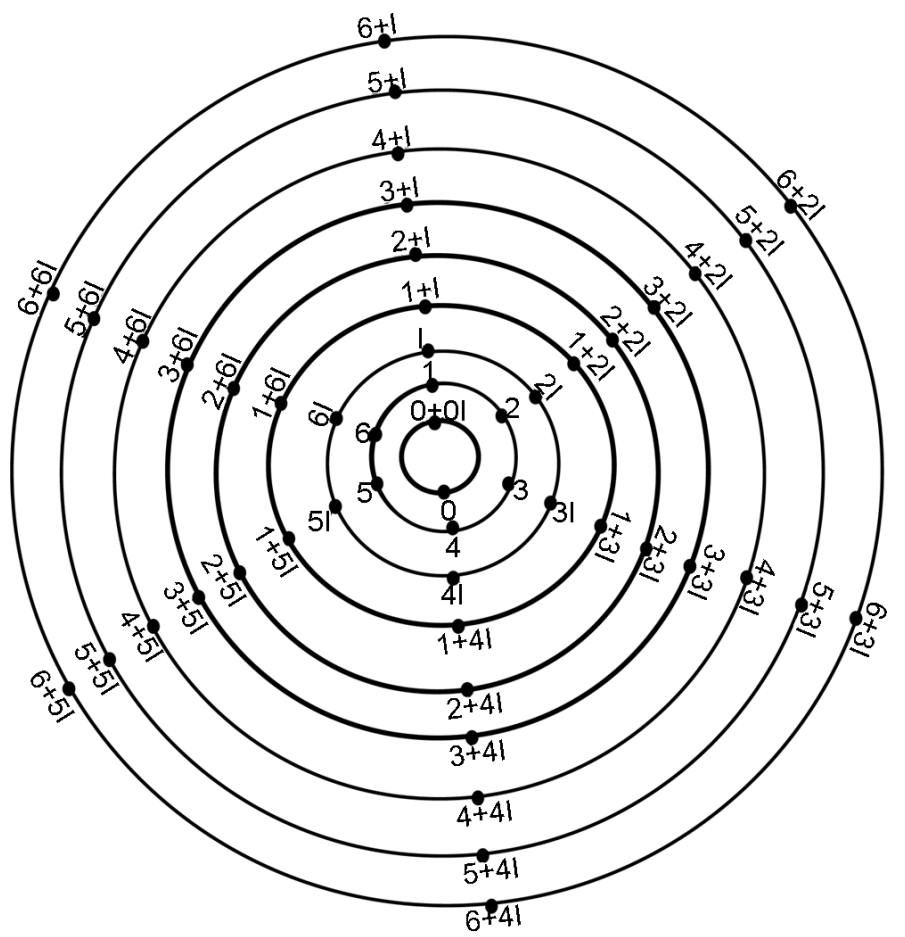

Figure 3. Cyclic neutrosophic viral graph.

The figure above is a cyclic representation of the neutrosophic group $(G)$. It depicts the connections between the nodes in order of their generation. Thus eight (8) cycles were formed with six (6) elements each together with the terminating cycle for the null neutrosophic or identity as shown in Figure 3.

This is indicating that circulation of viral infection within the same class is faster than its interconnectivity. And from the large circle we can see that, connection from node $6+I$ to node $6+2 I$ indicates the reversal since the graph is undirected, which shows involution. Moreover the distance between these three nodes $6+6 I, 6+I, 6+2 I$ remains equal when considering the shortest possible routes.

\section{Conclusions}

In this paper we represented an entire epidemic population with integer modulo 7, i.e., $\left(Z_{7}\right)$ and realize that $\left(Z_{7}+\right)$ is a group. Then we transform this group $\left(Z_{7}+\right)$ into a neutrosophic group $N(G)$, by defining $N(G)=\left\{\left\langle Z_{7} \cup I\right\rangle\right.$, '+'modulo 7$\}=\left\{a+b I / a, b\left(Z_{7}\right)\right\}$. We found that this is a special type of neutrosophic group which is also a group and in particular a cyclic group and having a set of generators each, which partitioned $N(G)$ into classes of subset which are themselves groups when the identity $0+0 I$ is embedded to each subset.

The results obtained is being converted into neutrosophic graph $\varnothing(V, E)$ with elements of $N(G)$ forming the vertices $V$ and $E$ is an arc determined by defining a suitable homeomorphisms between the groups $\left(Z_{7}+\right)$ and $N(G)$ as shown above. 


\section{References}

[1] Kermack, W.O. and McKendrick, A.G. (1927) A Contribution to the Mathematical Theory of Epidemics. Proceedings of the Royal Society Lond. A, 115, 700-721. https://doi.org/10.1098/rspa.1927.0118

[2] Brauer, A., Haug, G.H., Dulski, P., Sigman, D.M. and Negendank, J.F.W. (2008) An Abrupt Wind Shift in Western Europe at the Onset of the Younger Dryas Cold Period. Nature Geoscience, 1, 520-523. https://doi.org/10.1038/ngeo263

[3] Smarandache, F. (2014) Neutrosophic logic and set. http://fs.gallup.unme.edu/neutrosophy

[4] Vasantha Kandasamy, W.B and Smarandache, F. (2015) Neutrosophic Graphs, a New Dimension to Graph Theory. EuropaNova, USA.

[5] Feinleib, M. (2001) A Dictionary of Epidemiology. In: John, M. and Last, R.A. Eds., 4th Edition, Chicago, USA, 93-101.

[6] Kramer, et al. (2010) Principles of Infectious Disease Epidemiology. Modern Infectious Disease Epidemiology, Springer, New York. https://doi.org/10.1007/978-0-387-93835-6

[7] Claude, B., Perrin, D. and Ruskin, H.J. (2009) Considerations for a Social and Geographical Framework for Agent-Based Epidemics. International Conference Computational Aspects of Social Networks CASON, 9, 149-154.

[8] Nelson, K.E. (2006) Infectious Disease Epidemiology. Theory and Practice, Jones \& Bartlett Publishers.

[9] Vasantha Kandasamy, W.B., Smarandache, F. and Ilanthenral, K. (2005) Introduction to Linear Bialgebra, Hexis, Phoenix.

[10] Vasantha Kandasamy, W.B., and Florentin S. (2005) Basic Neutrosophic Algebraic Structures and Their Applications to Fuzzy and Neutrosophic Models, Hexis, Church Rock.

[11] Cayley, A. (1952) Collected Mathematical Papers. Cambridge University press. https://doi.org/10.1017/CBO9780511703768

[12] Michael, J.D. and Michael, R.F. (1991) Algebraic Constructions of Efficient Broadcast Netwoks. Los Alamos National Laboratorym New Mexico.

[13] Michael, J.D. (1991) Algebraic Methods for Efficient Network Constructions. University of Victoria, Idaho, 19-25.

[14] Bretto, A. and Gillibert, L. (2004) Graphical and Computational Representation of Groups. In: Proceedings of ICCS, In: LNCS, Springer-Verlag, Berlin, 343-350.

https://doi.org/10.1007/978-3-540-25944-2_44 
Submit or recommend next manuscript to SCIRP and we will provide best service for you:

Accepting pre-submission inquiries through Email, Facebook, LinkedIn, Twitter, etc. A wide selection of journals (inclusive of 9 subjects, more than 200 journals)

Providing 24-hour high-quality service

User-friendly online submission system

Fair and swift peer-review system

Efficient typesetting and proofreading procedure

Display of the result of downloads and visits, as well as the number of cited articles Maximum dissemination of your research work

Submit your manuscript at: http://papersubmission.scirp.org/

Or contact ojdm@scirp.org 